\title{
Deep Learning Algorithm for Urban Feature Extraction using SAR Data
}

\author{
${ }^{1}$ Nikunj Pithva , ${ }^{1}$ A. Vyas , ${ }^{1}$ Darshna Rawal , ${ }^{1}$ V. Nizalapur,${ }^{2}$ G. Jain, ${ }^{2}$ A. Das \\ ${ }^{I}$ Center for Applied Geomatics, CRDF, CEPT University, Ahmedabad 380009 \\ ${ }^{2}$ Space Applications Centre (ISRO), Ahmedabad 380015 \\ *Corresponding author e-mail: pitnik44@gmail.com
}

\begin{abstract}
Commission TCIII-Remote Sensing
KEY WORDS: SAR, Deep Learning, Convolution Neural Network, Feature Extraction, Segmentation,
\end{abstract}

\begin{abstract}
:
This paper aims to discusses the extraction of urban features from airborne NISAR (NASA-ISRO SAR) data using deep learning algorithm for a part of Ahmedabad City. NISAR data is acquired in two wavelength bands (L and S) in hybrid polarization i.e., RH and RV. This study has used level two data viz., amplitude data. Pre-processing of NISAR data in L and S wavelength bands was carried out by using MIDAS, software developed and provided by the Space Applications Centre. Pre-processing viz., Speckle suppression using different filters in varying window sizes, radiometric and geometric calibration was performed. Variation of backscattering coefficient (Sigma- nought) in different wavelengths and polarizations for different land use features were analysed. NISAR data in conjunction with LISS $4(5.8 \mathrm{~m}$ resolution) data is subjected to different fusion techniques. Qualitative and Quantitative analysis was carried out and Gram Schmidt technique was chosen for further analysis. Segmentation was performed to achieve better analysis of the fused image and the amplitude image. Lastly, a deep learning architecture was developed for the automatic classification of the image, and the Convolution Neural Network model was designed using mobile net and the regularization techniques. Deep learning architecture in conjunction with e-cognition developer was used for extracting urban features.
\end{abstract}

\section{INTRODUCTION}

Synthetic aperture radar (SAR) is an active remote sensing technology that can gather ground information at any time and under any circumstances. In SAR imaging processing, the coherent interaction between elementary scatterers on the ground and the electromagnetic waves leads to a multiplicative noise, known as speckle, affecting the SAR images. It is essential to monitor urban change at spatial and temporal scales to monitor the changes in cities and their impact on natural assets and environmental systems. Analyse this urban feature using deep learning such as deep neural networks and the architecture has extracted the urban area. Deep learning-based algorithms have exceeded conventional algorithms in terms of performance by a significant limit.(Wang et al. 2019) Successful management policies include revised and accurate information representing the current urban status. Present reporting methods are inefficient and incapable of holding the information up to date. For better identification of the feature and to extract information remote sensing is used. Identification and monitoring of urban areas through airborne and spaceborne missions is the best approach. Synthetic aperture radar (SAR) has long been accepted in many remote sensing systems as an effective tool for urban data analysis due to its all-weather capability and depicting geometrical properties of the target. The deep learning algorithm is being used for the classification of the images and the backscatter signatures library.(Pottier 2011)

\section{STUDY AREA AND DATA USED}

The selected study area was Ahmedabad city one slice of bopal region. This is the growing city of Gujarat. The city Ahmedabad selected to characterize its urban form. Under the city corporation jurisdiction, the extent of the city is 190.84 square kilometres. The river Sabarmati flows inside the city Ahmedabad. The city is renowned for its textile industries. It is observing the massive rate of urban sprawl towards all directions around $20 \mathrm{~km}$ from its centre.

NISAR data acquire in two-wavelength, L, and S. It has two different polarizations. In this circular polarization data is transmitted in circular polarization. (R). It received in linear polarization horizontal and vertical.

\begin{tabular}{|l|l|}
\hline \multicolumn{2}{|c|}{ AIR BONE NISAR Data Specifications } \\
\hline Date of Acquisition & $16-$ Jun-2017 \\
\hline No. Scans & 8587 \\
\hline No. Pixels & 2683 \\
\hline Near Incidence Angle & 18.02 \\
\hline Far Incidence Angle & 48.7 \\
\hline No. of Freq. Bands & L and S \\
\hline SAR Mode & CP \\
\hline No. of Polarizations & RH, RV \\
\hline Sensor Orientation & Left \\
\hline Look Angle Beam No. & 39.1 \\
\hline Frequency L band & $1250 \mathrm{MHz}$ \\
\hline Frequency S band & $3200 \mathrm{MHz}$ \\
\hline
\end{tabular}

Table 1. Data Specifications

\section{METHODOLOGY}

The methodology comprises of data preparation, analysis of the data and modelling. It is implemented in four phases: Literature survey, Selection of data and study area; Pre-processing of SAR data; Fusion techniques and Segmentation; Deep learning algorithm and Feature extraction. In the first phase understanding SAR, data and its basics for better implementation. Various manuscripts and Reports were referred to understand different approaches used to implement in the present study. In second phase, Pre-processing of NISAR data in $\mathrm{L}$ and $\mathrm{S}$ wavelength bands was carried out by using MIDAS, 
software developed by SAC. Pre-processing viz., Speckle suppression using different filters in varying window sizes, radiometric and geometric calibration was performed. After the pre-processing, Variation of backscattering coefficient (Sigmanought) in different wavelengths and polarizations for different features were analysed. For a better understanding and enhancement of the functionality, NISAR data was fused with LISS 4 MX data. Specific fusion techniques were performed and analysed, such as HIS (Intensity Hue Saturation), Borvey, Ehlers Resolution Merges, PCA (Principal Component Analysis) and Gram Schmidt (GS). Appropriate technique (GS) was selected from the qualitative and quantitative analysis. In the last stage, Segmentation was performed for better analysis of the fused image and the amplitude image. A deep learning architecture was developed for the automatic classification of the image, and the Convolution neural network model was designed using mobile net and the regularization techniques. Deep learning architecture in conjunction with e-cognition developer was used for extracting urban features.

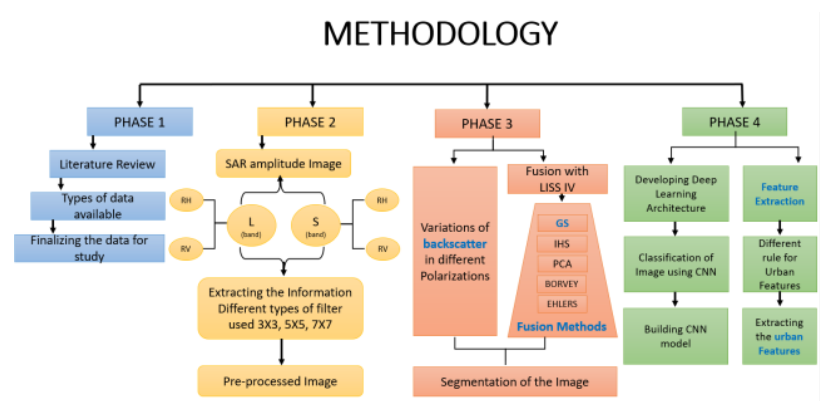

Figure 1. Four Phases of Methodology

\subsection{Data Pre-processing}

Aim of this research to extract urban features from the NISAR data. Pre-processing of the data and generation of the primary result was performed on Microwave Data Analysis Software (MIDAS) software. MIDAS is in-house developed SAR data processing software. This software is developed in Advanced Microwave and Hyperspectral Techniques Development Group (AMHTDG)/EPSA (Techniques ,2018). To start with, speckle suppression was carried out using different types of filters in different window sizes. From the different window sizes, we observed different results and one window size is fixed for our data set and further analysed. For the removing noise from the data, we used ten different types of speckle suppression filters and window sizes for identifying which filter has suited the best of the pre-processing of the data. The appropriate speckle suppression filter is chosen based on SSI value which is lower than a filter. From the trial-and-error method, we found out the Enhanced Lee with $3 \times 3$ window size is appropriate speckle suppression filter for this study.
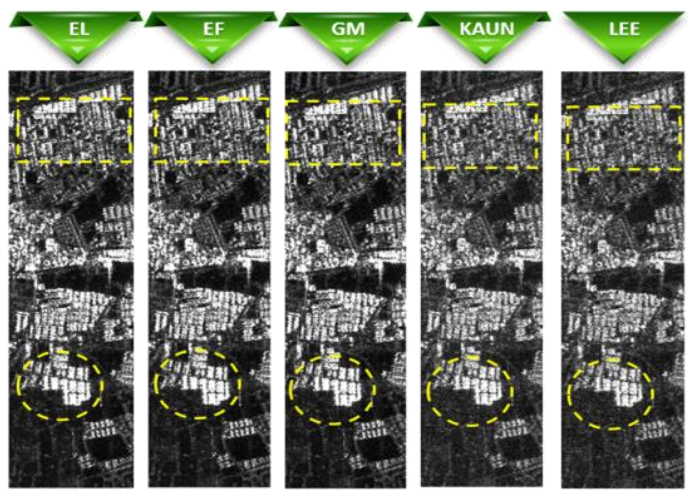

Figure 2. Result of Different filters

\begin{tabular}{|c|c|c|c|c|c|c|}
\hline No. & $\begin{array}{ll}\text { TYPE } & \text { OF } \\
\text { FILTER } & \end{array}$ & MEAN & $\begin{array}{l}\text { ST. } \\
\text { DEVIATION }\end{array}$ & MSE & SSI SD & $\begin{array}{l}\text { SSI } \\
\text { MEAN }\end{array}$ \\
\hline 0 & $\begin{array}{c}\text { WITHOUT } \\
\text { FILTER }\end{array}$ & 113.4654 & 164.4284 & - & - & \\
\hline 1 & Enhanced Lee & 113.0274 & 142.6890 & 2239.6840 & 0.7499 & 1.0310 \\
\hline 2 & Gamma Map & 113.3155 & 142.8636 & 2409.8944 & 0.8747 & 1.0372 \\
\hline 3 & Enhanced Forst & 113.3207 & 143.1069 & 2392.6385 & 0.8697 & 1.1182 \\
\hline 4 & Kaun & 107.0552 & 140.8479 & 12699.7229 & 1.0007 & 1.1045 \\
\hline 5 & Lee & 107.5900 & 145.4212 & 15747.3988 & 1.068 & 1.13666 \\
\hline 6 & Mean & 113.4655 & 143.3809 & 2476.1221 & 1.0054 & 1.039 \\
\hline 7 & Median & 108.8976 & 137.1858 & 2576.4321 & 0.7860 & 1.075 \\
\hline 8 & Refined Lee & 72.7882 & 83.6689 & 1616.3395 & 1.1238 & 0.7272 \\
\hline 9 & Sigma & 113.4653 & 143.3811 & 2476.1221 & 0.993 & 1.1217 \\
\hline 10 & SARAD & 113.8087 & 131.5047 & 4967.5609 & 1.4409 & 1.1036 \\
\hline
\end{tabular}

Table 2. Evaluation of filters

\subsection{Back Scatter co-efficient}

Area of the isotropic target which will reflect (backscatter) power the same as that being received by the system, corresponding to a given transmit power. The normalized measured of the radar return from a distributed target is called the "Backscatter Co-efficient". Scattering by targets = returns in multiple directions.(Pottier 2011)

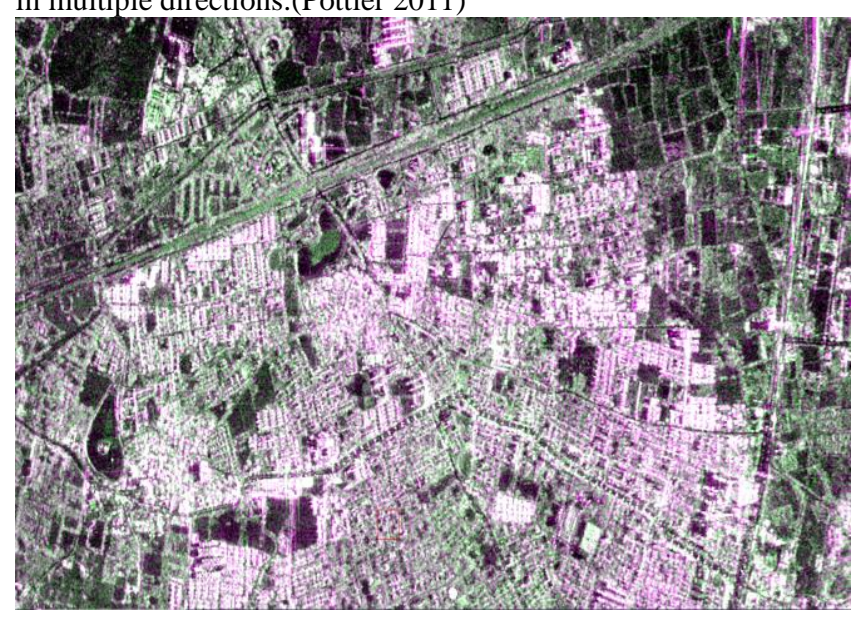

Figure 3. Pre-processed Image 


\subsubsection{Mean back scatter co-efficient}

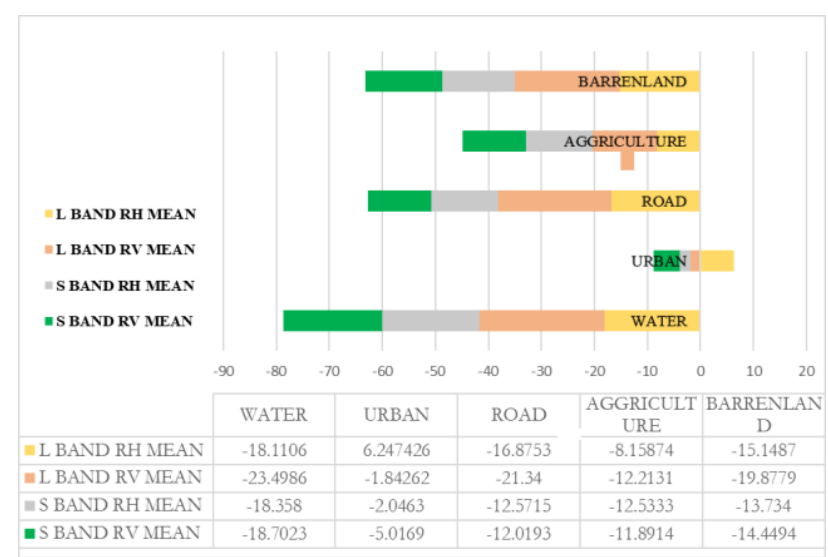

Figure 4. Mean of Backscatter

As per the graph, values are different for different types of features. For the same wavelength in RH in two-band L and S. As per the graph, backscattering coefficient values of different land use are observed as urban area values range from -5 to +5 $\mathrm{dB}$; Road values range from -12 to $-20 \mathrm{~dB}$; Water bodies range from -18 to $-23 \mathrm{~dB}$; Barren land lies between -13 to $-19 \mathrm{~dB}$ and Agriculture area values lie between -8 to $-11 \mathrm{~dB}$.

\subsubsection{Variation of Sigma naught for Different Features}

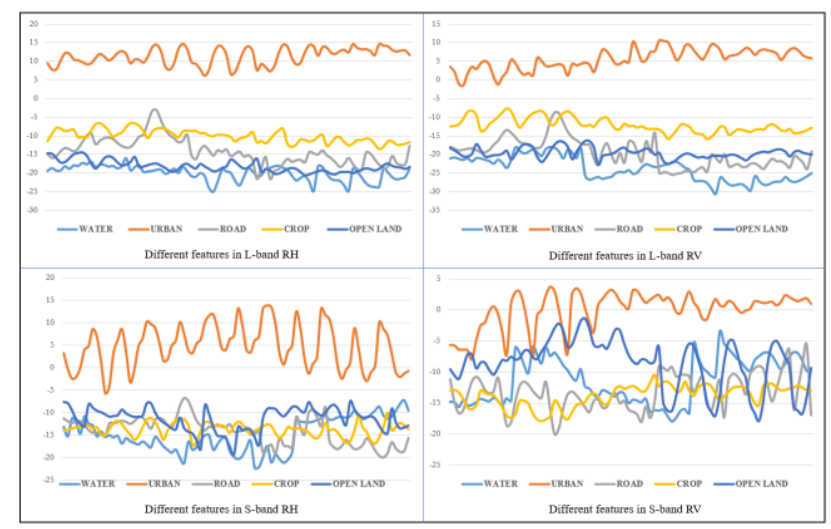

Figure 5. Different features in $\mathrm{L}$ and $\mathrm{S}$ band RH \& RV

Variation of sigma naught value o different features is analysed for $\mathrm{L}$ and $\mathrm{S}$ wavelength in horizontal (RH) and Vertical (RV) polarizations. RH urban values are in positive where RV some values are mixed with other feature, so it is in the negative. Like with different features values are different in both the band and polarization also. As per the analysis, we observed that $\mathrm{L}$ band RH has good results than the others. Therefore, that is useful for the identification of urban features. There is an overlap of backscatter coefficient values between the different features hence, they may not be discriminated accurately.

\subsubsection{Variation of Sigma naught for Urban Features}

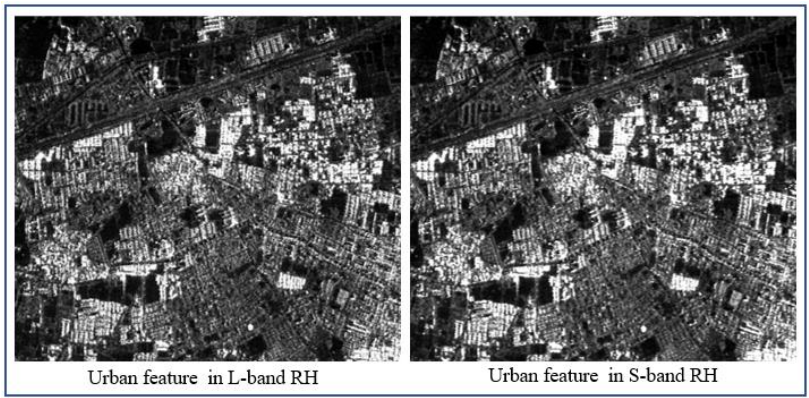

Figure 6. Different features in $\mathrm{L}$ and $\mathrm{S}$ band $\mathrm{RH} \& \mathrm{RV}$

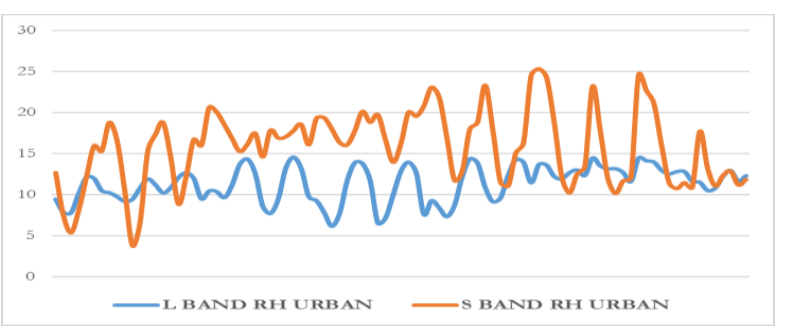

Figure 7. Different urban features in L and S band RH

$\mathrm{L}$ and S-band characteristics are different so after the process data, both bands are $\mathrm{RH}$ value is comparing with each other in the urban features. As per the graph is it has clearly shown that the result of urban features is better in the $\mathrm{L}$ band than the Sband.

\subsection{Analysis on Processed Image}

After the processed image, our goal is to extract urban features. In the analysis, we observed that built-up in different orientation has different backscatter values. In order to analyse, different Region of Interests (ROI's) were selected for different building orientations as shown in the image. Variation of backscatter with respect to orientation of the building is carried out.

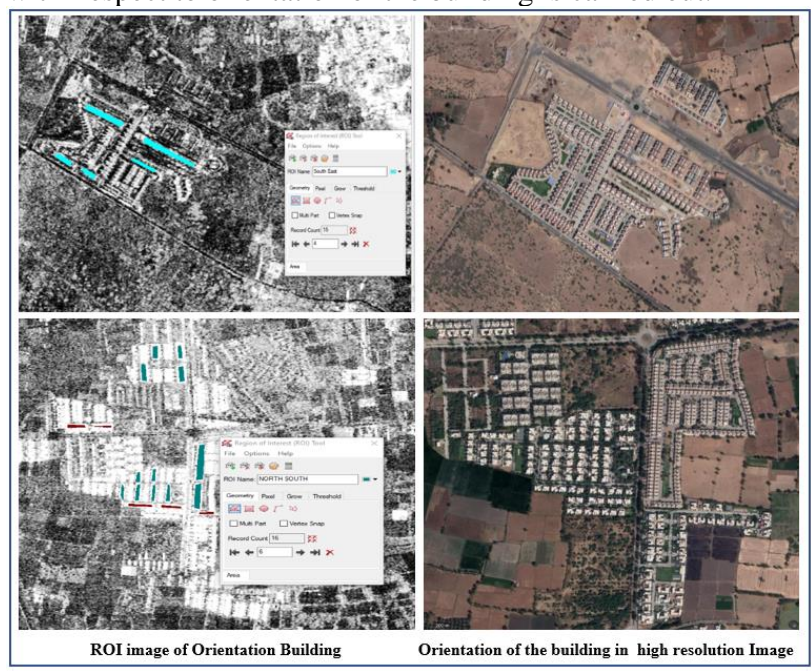

Figure 8. Different Orientation of Buildings. 


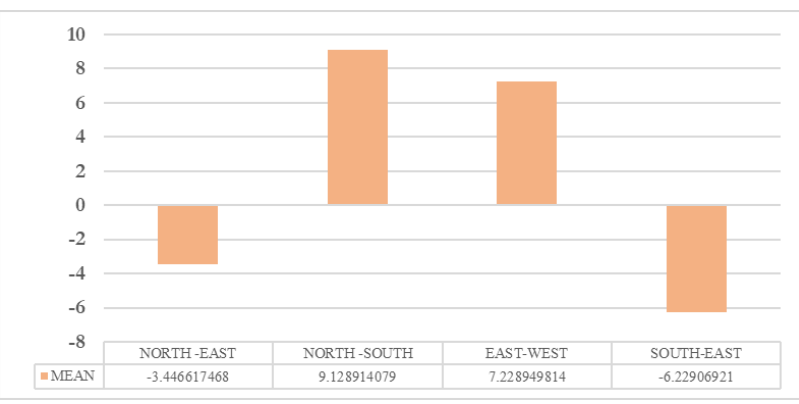

Figure 9. Different Orientation of Buildings in Graph.

The backscatter value differs for high-rise and low-rise buildings. ROI took for high-rise and low-rise buildings; a reflection of the high-rise building is more than the low-rise buildings. Same as backscatter value is different for the highly dense urban rea and sparsely dense urban areas as shown in the fig sparsely dense has low value compared to highly dense urban areas.

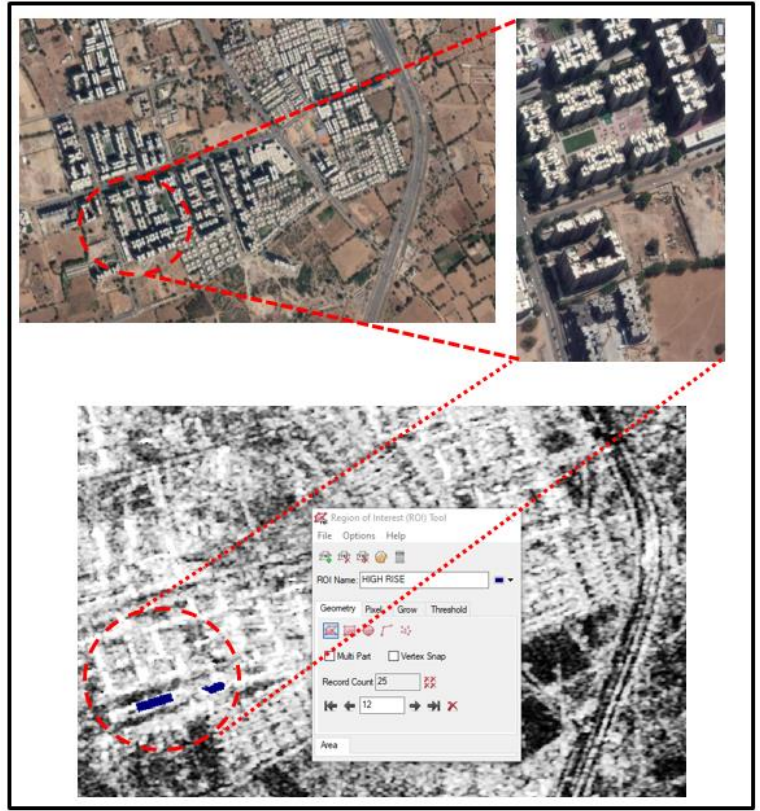

Figure 10. High- Rise and Low-rise building and the ROI's.

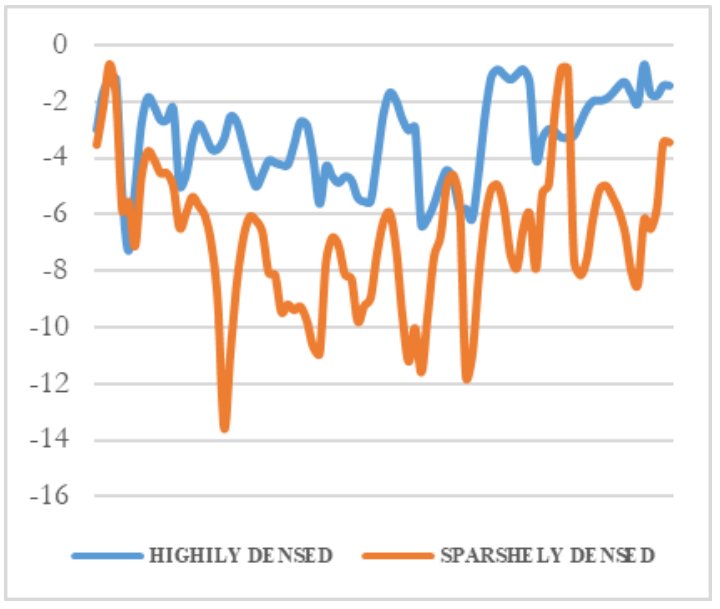

Figure 11. Highly dense and Sparsely dense backscatter.

\subsection{Image Fusion}

The image fusion process allows merging multispectral imagery of relatively low spatial resolution with other co-registered panchromatic images of relatively high resolution. However, for this research, we are using fusion, not for high resolution but we use fusion for feature enhancement. Available literature suggests several approaches to perform the fusion. The commonly used process known as Principal Component Analysis (PCA), Modified Borvey, Intensity Hue Saturation (IHS), Ehlers resolution merge, and Gram Schmidt (GS).For the evaluation of the fusion, there are two techniques we are using in this research.(Jia et al. 2019)

\subsubsection{Qualitative Test}

From the visual interpretation, the fusion images are good. From the different methods, which fusion method has improved results compared to other methods? The interpreter analyses the tone, contrast, saturation, sharpness, and texture of the fused images.

\section{A) Borvey Method}

For the histogram, this method developed to gain the observed votarist in the high and low end. Three-band at a time are combined from multispectral scene.

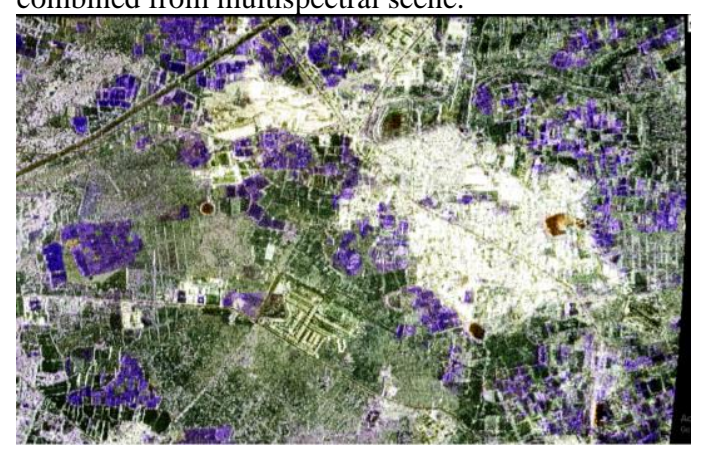

Figure 12. Borvey Fused Image.

\section{B) Intensity Hue Saturation(HIS) Method}

It Separates spatial (intensity) and spectral information from a standard RGB image. Preserves more spatial features and more required functional information with no color distortion. Only three bands involved in it.(Web 2018)

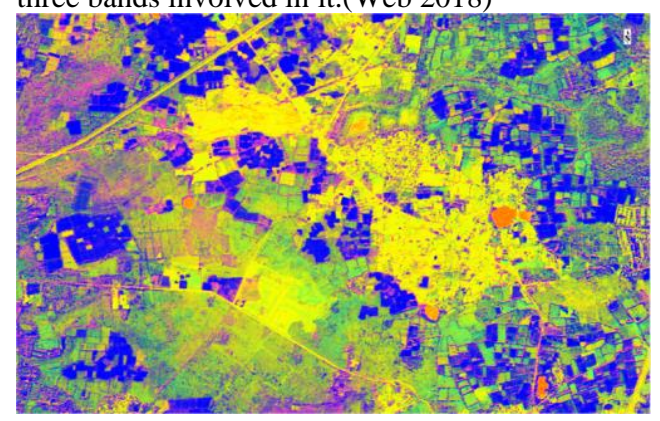

Figure 11. IHS Fused Image. 
C)

\section{Ehlers Resolution Merged Method}

It is based on N IHS transform coupled with Fourier domain filtering. To facilitate these demands two prerequisites have to be addressed. First color and spatial information have to be separated.(Bao et al. 2012)

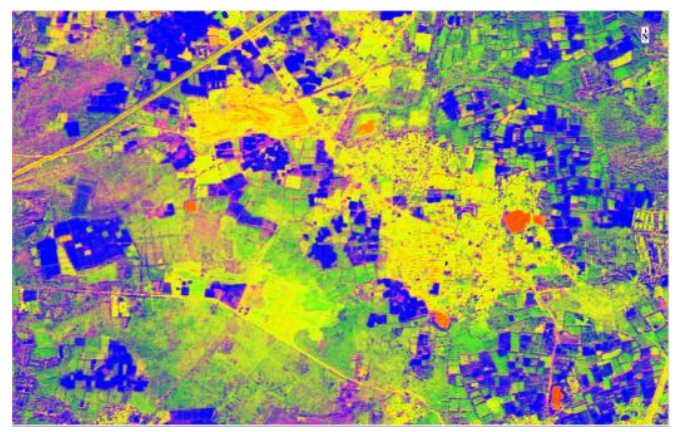

Figure 14. Ehlers Fused Image.

\section{D) Principal Component analysis method}

A statistical technique transforms a multivariate dataset of correlated variables into a dataset of the uncorrelated linear combination of the original variables. (Bao et al. 2012)

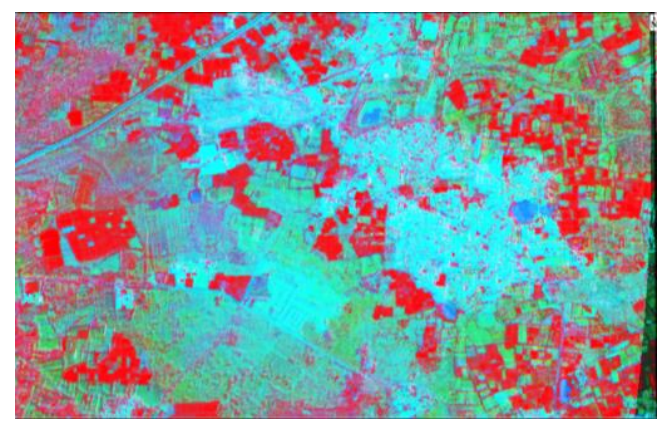

Figure 15. PCA Fused Image.

\section{E) Gram Schmidt Method}

The gram-Schmidt process transforms a set of vectors into a new set of orthogonal and linearly independent vectors.(Web 2018)

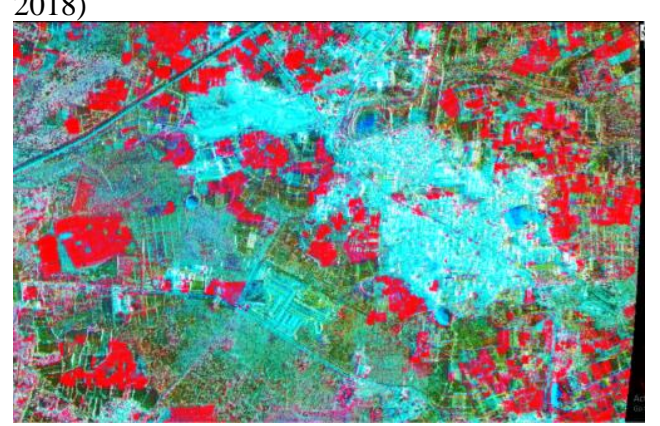

Figure 16. Gram Schmidt Fused Image.

\subsubsection{Quantitative Statistical Test}

\section{Spectral Evaluation:}

The bias of mean: The value is given relative to the mean value of the original image. The ideal value is zero.(Web 2018)

$$
\text { Bias }=\frac{M S_{\text {mean }}-\text { Fused }_{\text {mean }}}{M S_{\text {mean }}}
$$

\section{Spatial Evaluation:}

Entropy: If the entropy of the fused image is higher than parent image then it indicates

that the fused image contains more information.(Bao et al 2012)

\begin{tabular}{|c|c|c|c|c|}
\hline METHODS & MEAN & ST.DEV & ENTROPY & BIAS \\
\hline PCA & 372.7772 & 71.07881 & 3.606921 & 0.357324 \\
\hline Gram Schmidt & $\mathbf{3 0 3 . 3 6 8 8}$ & $\mathbf{1 0 2 . 5 2 9 9}$ & $\mathbf{4 . 3 6 8 7 3 1}$ & $\mathbf{0 . 2 5 3 3 6 7}$ \\
& & & & \\
\hline BORVEY & 101.3572 & 140.9065 & 0.445224 & 1.430566 \\
\hline IHS & 369.6045 & 45.90559 & 3.265826 & 0.346943 \\
\hline EHLERS & 681.1566 & 75.34437 & 3.506369 & 4.925797 \\
\hline
\end{tabular}

Table 3. Quantitative Statistical Test

As per the analysis, the mean and standard deviation values closer to those of the actual image value. Highest value obtained in Gram Schmidt fusion for Entropy. Same as lower value in Bias also obtained in the Gram Schmidt of this research Gram Schmidt method is highly take into consideration.

\subsection{Segmentation}

In this, it provides a detailed specification of different segmentation software. Later image segmentation applied using different image segmentation software on ENVI. Image segmentation parameter is defined by applying the trial-anderror approach and finally, the best segmentation result was drawn by making visual (assessment. Alternatively, consideration.) Quantitative quality assessment was made based on the visual observation besides a descriptive explanation of algorithms used by different segmentation tools was also included. The result achieved by applying multi-resolution segmentation.

\subsubsection{Image Segmentation: Multi resolution Segmentation}

Multiresolution segmentation is used to test the segmentation result. Scale parameter defined as 50 for image segmentation as well as the shape and colour properties assigned as 0.1 and 0.5 respectively. Overall segmentation quality is quite good, despite there are some over-segmentations and very few under segmentation. The major reason for the mismatch between the urban features edges polygon and the segmented result is because reference polygon was drawn much-generalized way represents the outline of the feature outline. 


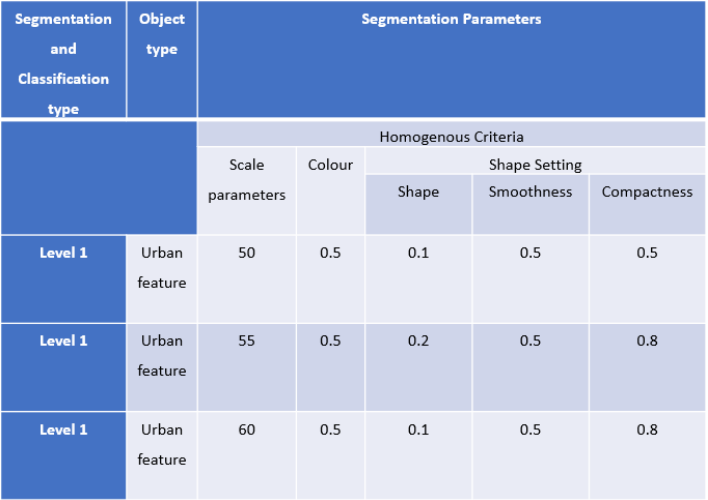

Table 4. Multi resolution Segmentation
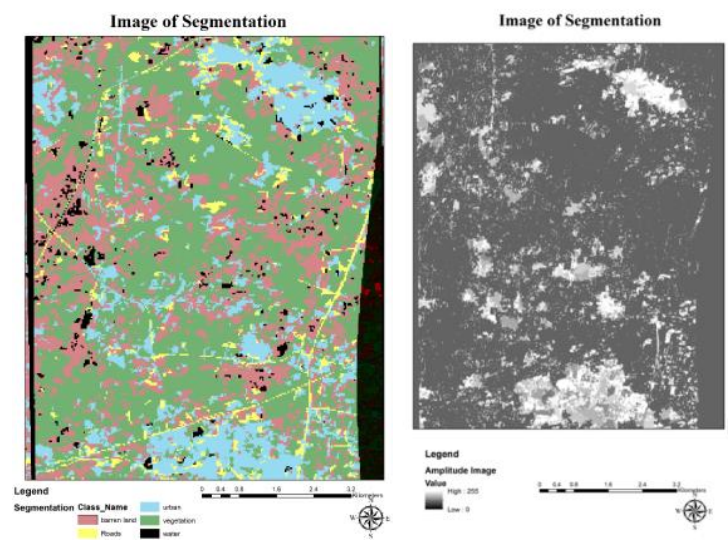

Figure 17. Fused and Amplitude Segmented Image

\subsection{Deep Learning Architecture}

$\mathrm{CNN}$ is a specialized kind of neural network for processing data that has a known, grid-like topology, such as time series (1D grid), image data (2D grid), etc. Build a $\mathrm{CNN}$, has 60 million parameters, and 650,000 neurons, consists of five convolutional layers. Typical $\mathrm{CNN}$ is a 5-layer architecture consist of convolution layer, pooling layer, and classification layer.(Wang et al. 2019)

\subsubsection{Diagram of Convolution neural Network}

Data Creation: After the fusion with the Liss-4 image, we have two different types of input for the classification of the image. One is amplitude RH image, and another is the fused image. In the first part, we have to create data for CNN. Using the deep learning algorithm tool, we sliced images into different window sizes. For this study, we take 256x 256, 350 x 350-window size taken into consideration. It takes an input file like JPEG, PNG, and Tiff formats. After the whole image sliced in particular window sizes then we have to label all the images. After that first part of the diagram is ready to input the images into the model.

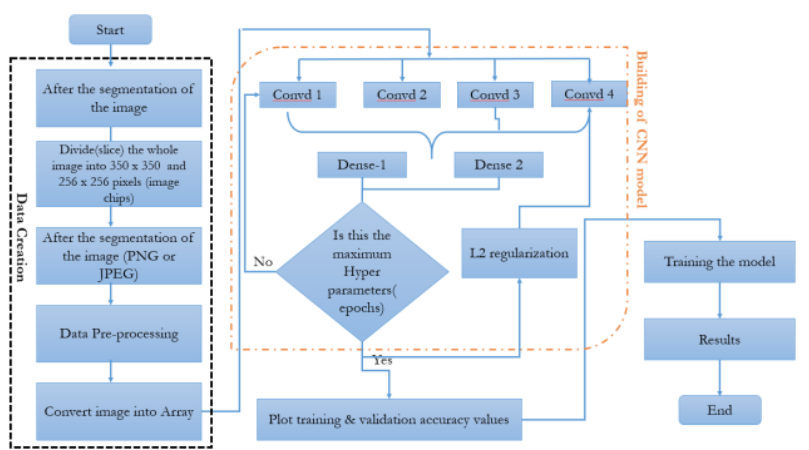

Figure 18. Flow chart of CNN.

Convert images into Array: After creating, the data for the first step is to input data in the model. A CSV file created as a label to understand the data. From the label and the images, model test and train for the further process. For understanding data to the machine, the images have to be converted into an array. Converted files then put as test and train data as per the requirement.

Building of CNN model: The whole model divided into four parts. Where the different matrix values are using to train the model and how the features in the images have. Then dense layer created and dropout measure for the model. For the increasing accuracy, it depends on its hyper-parameters (epochs or iteration). As the hyper-parameter increases the accuracy also increases. Regularization is used for further improvement.

Plot training and validation accuracy values: Applying the different hyper-parameter values we get different types of graphs and values for the training and accuracy of value as per how our model is training using the model.

Training and Testing the model: Now the deep learning model trained according to the convolution neural network layers. Therefore, we have to test the model on how it gives the classification of the image. For the different types of window sizes and no. for training data set used in the machine plays an important role in CNN.

\section{RESULTS AND DISCUSSION}

For target identification classes such as built-up, within water body and city, linear features like road and bridge, vegetated land, open field, and water body are taken to analyse and study. Also, certain linear features, such as roads and bridges, and trees, open fields, and water bodies.

\subsection{Result of CNN for fused Image ( $256 \mathbf{X} 256$ )}

For, the CNN applied on fused image for the classification because it can identify all the features in the image. There was a 256-X-256 window size with the approx. 1800 image chips used in this model. (Table 5) For a better result, more the dataset gives more accurate results. We used regularization model with different accuracy we get in running our model.

\begin{tabular}{|l|l|l|l|l|l|}
\hline No. & $\begin{array}{l}\text { Inputs of 256x256 } \\
\text { window size ( 1800) }\end{array}$ & $\begin{array}{l}\text { Regularize } \\
\text { Model }\end{array}$ & $\begin{array}{l}\text { Epochs } \\
\text { Used }\end{array}$ & Accuracy & Loss \\
\hline 1 & Fused Image & With & 5 & 0.8393 & 0.2247 \\
\hline 2 & Fused Image & With & 10 & 0.8143 & 0.2584 \\
\hline 3 & Fused Image & With & 20 & 0.8476 & 0.202 \\
\hline 4 & Fused Image & With & 50 & 0.8595 & 0.1062 \\
\hline 5 & Fused Image & With & 100 & 0.8601 & 0.0204 \\
\hline
\end{tabular}

Table 5. Fused Image Window Size (256 X 256) Used for the Training of Model 


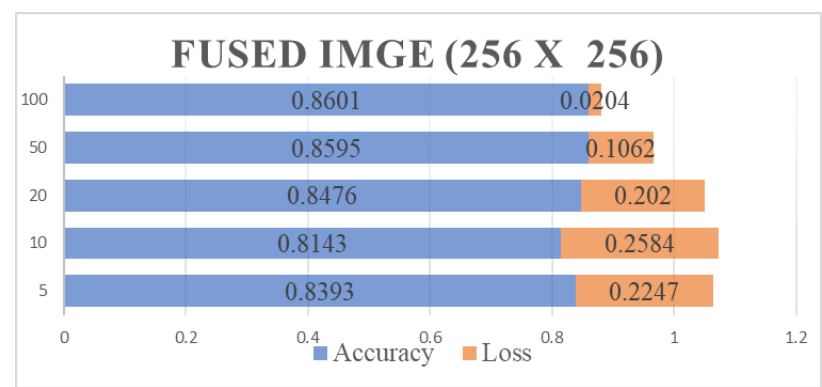

Figure 19. Graph of Fused Image (256 X 256).

As per the graph fig.20, with the different epochs, the accuracy and loss are also different for this window size we get approx. $80 \%$ of the accuracy and as shown in fig 20 , it identifies all the features within the image.

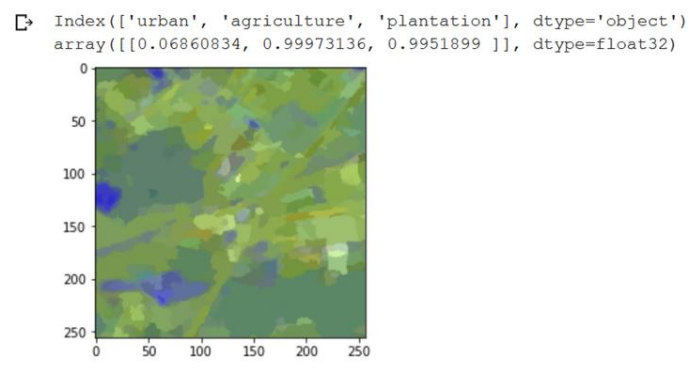

Figure 20. Result of 256 X 256 Fused Image.

\subsection{Result of CNN for fused Image ( 256 X 256)}

As per the window size is increasing the accuracy is decreasing but the feature classification is also better in this model. (Table 6)

\begin{tabular}{|l|l|l|l|l|l|}
\hline No. & $\begin{array}{l}\text { Inputs of } \\
\text { 350x350 window } \\
\text { size(931 ) }\end{array}$ & $\begin{array}{l}\text { Regularize } \\
\text { Model }\end{array}$ & $\begin{array}{l}\text { Epochs } \\
\text { Used }\end{array}$ & Accuraccy & Loss \\
\hline 1 & Fused Image & With & 5 & 0.8286 & 0.5734 \\
\hline 2 & Fused Image & With & 10 & 0.795 & 0.3104 \\
\hline 3 & Fused Image & With & 20 & 0.8357 & 0.2756 \\
\hline 4 & Fused Image & With & 50 & 0.8286 & 0.0662 \\
\hline 5 & Fused Image & With & 100 & 0.8071 & 0.012 \\
\hline & & & & & \\
\hline
\end{tabular}

Table 6. Fused Image Fused Image Window Size (256 X 256) Used for the Training of Model

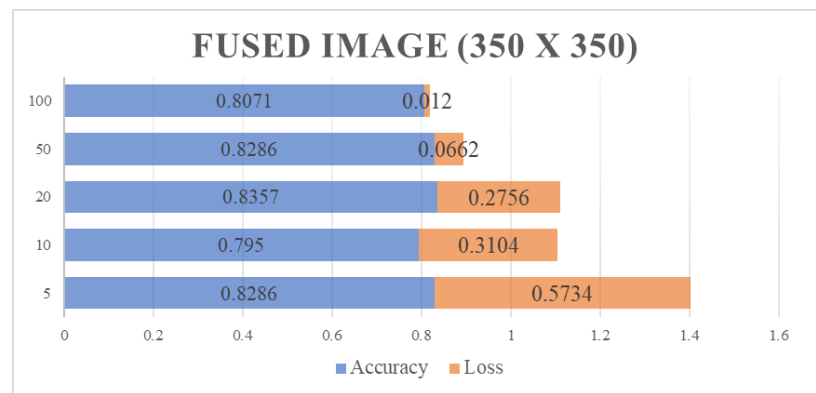

Figure 21. Graph of Fused Image ( 350 X 350).
Here, as shown in fig 22, the result obtained for agriculture and plantation classification is better as per the feature in the image so the model accuracy can improve with the use of different epochs.

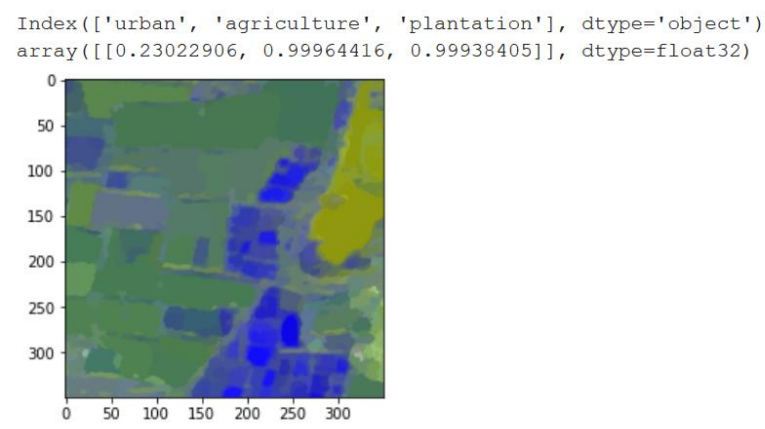

Figure 22. Result of 350X350 Fused Image.

\subsection{Result of CNN for amplitude Image ( 256 X 256)}

In this part, we are using the only urban feature for classification of the image because the amplitude image has given better classification for urban features other features are mixing. (Table 7).

\begin{tabular}{|l|l|l|l|l|l|}
\hline No & Inputs & $\begin{array}{l}\text { Regularize } \\
\text { Model }\end{array}$ & $\begin{array}{l}\text { Epochs } \\
\text { Used }\end{array}$ & $\begin{array}{l}\text { Accurac } \\
\text { cy }\end{array}$ & Loss \\
\hline 1 & Amplitude Image & With & 5 & 0.5979 & 0.705 \\
\hline 2 & Amplitude Image & With & 10 & 0.6424 & 0.5067 \\
\hline 3 & Segmented Image & With & 20 & 0.6825 & 0.1547 \\
\hline 4 & Segmented Image & With & 50 & 0.709 & 0.0689 \\
\hline 5 & Segmented Image & With & 100 & 0.7302 & 0.0246 \\
\hline
\end{tabular}

Table 7. Window Size ( 256 X 256) Used for the Training of Model

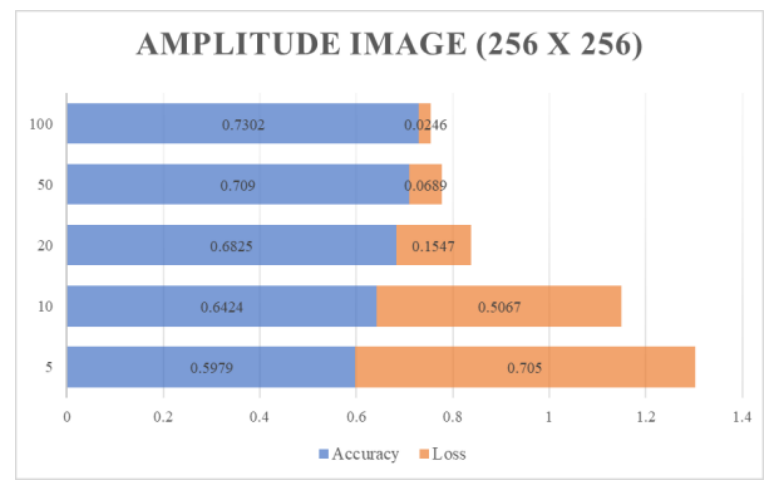

Figure 23. Graph of Amplitude Image (256 X 256).

As per fig.24, the model clearly shows that the train and validation data model are not overfitting. For the amplitude image, different epochs give different results as shown in the graph. The graph shows the values of model accuracy and the loss with the changes in epochs. After increasing the epoch, the loss is decreases and the accuracy is increases. In the model, 
regularization is very helpful for the better results and classification of the images. (Fig. 25)

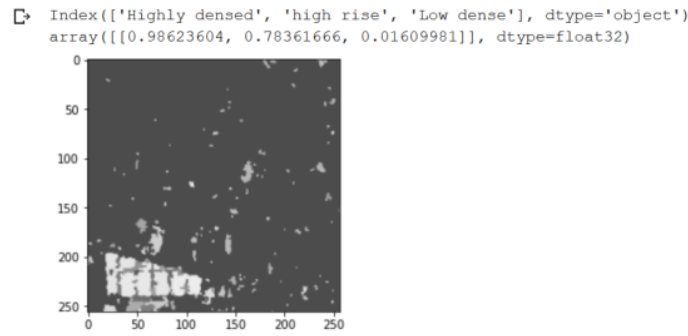

Figure 24. Result of 256 X 256 Amplitude Image.

\subsection{Result of CNN for amplitude Image (350 X 350)}

As the window size increasing the training samples are also, decreasing hence the accuracy is low and the loss is higher compared to the 256-window size. (Table 8)

\begin{tabular}{|l|l|l|l|l|l|}
\hline No. & $\begin{array}{l}\text { Inputs of } \\
\mathbf{3 5 0 x 3 5 0} \text { window size } \\
\mathbf{( 2 2 9 )}\end{array}$ & $\begin{array}{l}\text { Regularize } \\
\text { Model }\end{array}$ & $\begin{array}{l}\text { Epochs } \\
\text { Used }\end{array}$ & Accuracy & Loss \\
\hline 1 & Amplitude Image & With & 5 & 0.5619 & 0.6533 \\
\hline 2 & Amplitude Image & With & 10 & 0.4286 & 0.5246 \\
\hline 3 & Amplitude Image & With & 20 & 0.4286 & 0.1943 \\
\hline 4 & Amplitude Image & With & 50 & 0.5574 & 0.1018 \\
\hline 5 & Amplitude Image & With & 100 & 0.6667 & 0.048 \\
\hline & & & & & \\
\hline
\end{tabular}

Table 8. Fused Image Window Size ( 350 X 350) Used for the Training of Model

\section{AMPLITUDE IMAGE(350 X 350)}

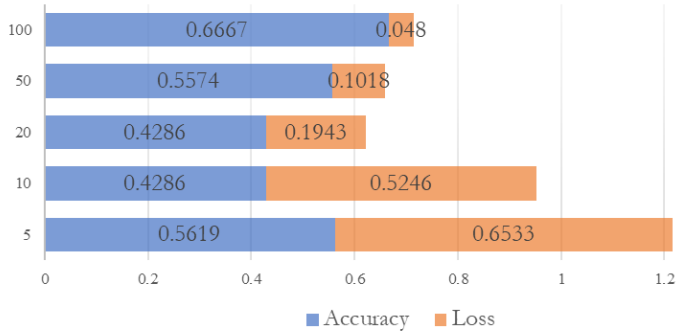

Figure 25. Graph of Amplitude Image (350X 350).

For this model, we achieved $0.66 \%$ of accuracy and $0.048 \%$ (as shown in Fig.26) of loss where the loss in small but accuracy as some of the features like highly dense feature classification is overfitting in this model. Also, for other feature like the low dense area, it identifies clearly. (Fig.27).

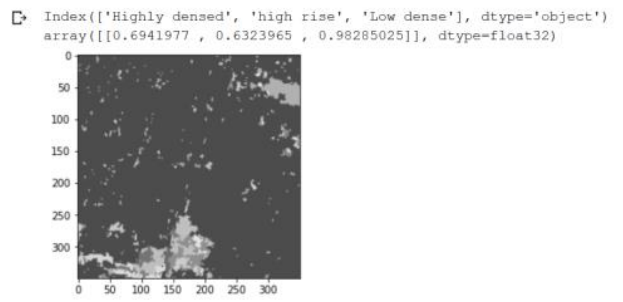

Figure 26. Result of 350 X 350 Amplitude Image.

\subsection{Extracting Urban features from the Image}

Using the e-cognition developer for different features assigns the class to each feature. In the first part after the final image, we have to first segment the image for the identification of different features from the image and assign the class to each feature for the classification of the image. Then the feature was selected using the different backscatter values. After selecting the feature from the value, the polygons are merged with the same feature. For the removal of the small polygons and those are not lies in the urban features has to remove from the image using the geometry the area pixel is used. After the removal, the neighbouring urban polygons were merged. Then the features were ready to extract from the image. As per the different formats, we extract the features. As shown in fig 49, from the amplitude image we extract the urban features.

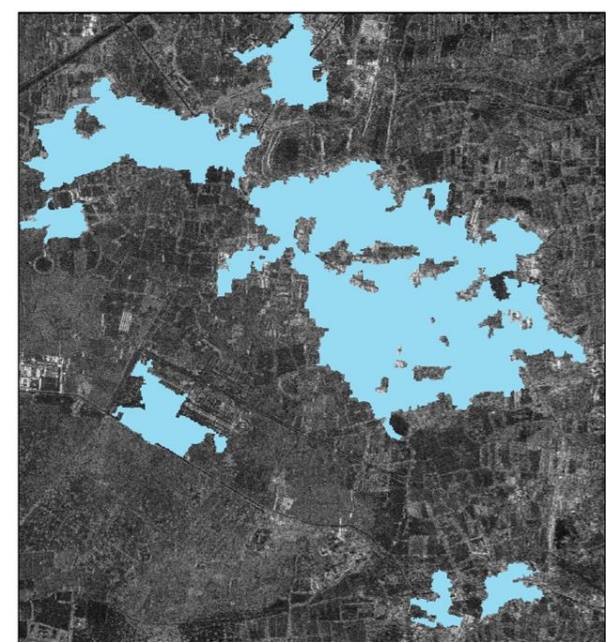

Figure 27. Feature Extraction form the amplitude Image.

As we can compare the result with the fused image, (as shown in Fig.28) that in the fused image we can identify all the features. In the amplitude image, we can identify the urban feature only, besides, we can also identify the Highly dense, Low dense and high rise building from the image and Extract that feature from the image as shown in the fig.

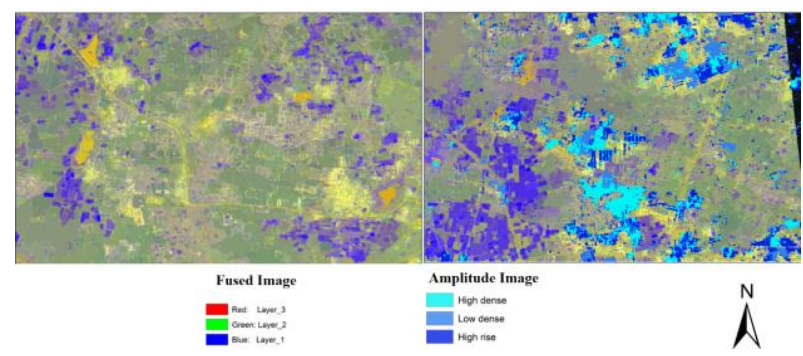

Figure 28. Fused and Amplitude Image feature extraction.

\section{CONCLUSION AND FUTURE SCOPE}

The present study addressed the potential of airborne NISAR data to discriminate against the land cover classes emphasizing the urban area. Pre-processing of NISAR data was carried out and it is observed that Enhanced Lee filter with window size $3 \mathrm{X} 3$ is suitable for the present study. Different type of fusion techniques was used as SAR and optical data give complementary information about the target. Based on qualitative and quantitative analysis, fused data of NISAR and 
L4 MX using Gram- Schmitt techniques were found suitable for the study and further analysed. For automatic classification of the image, a deep learning architecture was developed, and Convolution neural network model was built with the use of mobile net. In model training and testing, labelled data for the model has been created and using different types of hyperactive parameters. Classification accuracy of $80.67 \%$ and $70.81 \%$ was achieved for fused and amplitude images, respectively. We also observed that with the increase in no. of Epochs i.e., one of the hyper-parameters; the accuracy is increased, and loss is decreasing. Deep learning architecture in conjunction with ecognition developer has been used for extracting urban features by applying different types of the rule set for each feature and assigning classes. Segmentation of fused image was able to discriminate different land cover classes viz., Urban, vegetation, open land, water, roads while segmentation of RH amplitude data was able to discriminate different built-up forms viz., High rise, high dense and sparse dense built-up area.

This research has a vast scope in the future. In the present study, the identification of urban polygons was carried out using CNN. However, with the availability of high-resolution data, identification of slums, industrial areas, residential areas, etc within the urban area can also be done accurately. Urban growth monitoring, change detection, and prediction can be addressed more accurately as per today's need using deep learning. By using more no. of datasets for automation in the classification of images, the accuracy of more than $90 \%$ can also be achieved.

\section{ACKNOWLEDGEMENTS}

This study has been executed under Announcement of Opportunity $\mathrm{L}$ and $\mathrm{S}$ band SAR programme of SAC, ISRO. It is a part of SAC-CEPT collaborative project "SAR Remote Sensing of Urban area". Authors acknowledge anonymous reviewers for their constructive comments.

\section{REFERENCES}

Anon. 2006. "What Is Radar?" IEEE Aerospace and Electronic Systems Magazine 21(8):13-15.

Anon. n.d. "(No Title)." Retrieved May 6, 2020a (http://www.gecj.ac.in/download/RS_GIS model paper.pdf).

Anon. n.d. "Chair for Remote Sensing." Retrieved May 7, 2020b (https://www.geographie.uni-jena.de/eo.html).

Anon. n.d. "Classification of Polarization." Retrieved May 6, 2020c astr.gsu.edu/hbase/phyopt/polclas.html). (http://hyperphysics.phy-

Anon. n.d. "Radar Enhanced Frost Filter." Retrieved May 6, 2020d (https://www.pcigeomatics.com/geomaticahelp/concepts/orthoengine_c/Chapter_823.html).

Anon. n.d. "Radar Enhanced Lee Filter."

Anon. n.d. "Remote Sensing Technologies | Geospatial Data and Imagery | L3Harris Geospatial." Retrieved May 6, 2020 (https://www.harrisgeospatial.com/).

Bao, Chunhong, Guoman Huang, and Shucheng Yang. 2012. "APPLICATION OF FUSION WITH SAR AND OPTICAL IMAGES IN LAND USE." XXXIX(September):11-14.

Camargo, Flávio F., Edson E. Sano, Cláudia M. Almeida, José C. Mura, and Tati Almeida. 2019. "A Comparative Assessment of Machine-Learning Techniques for Land Use and Land Cover Classification of the Brazilian Tropical Savanna Using ALOS-2/PALSAR-2
Polarimetric Images.” Remote Sensing 11(13).

Dasari, Kiran, L. Anjaneyulu, P. V. Jayasri, and A. V. V. Prasad. 2016. "Importance of Speckle Filtering in Image Classification of SAR Data." 2015 International Conference on Microwave, Optical and Communication Engineering, ICMOCE 2015 (August 2016):349-52.

Furukawa, Hidetoshi. 2017. "Deep Learning for Target Classification from SAR Imagery: Data Augmentation and Translation Invariance." 13-17.

Hasni, Anas, Majdoulayne Hanifi, Chaimae Anibou, and Mohamed Nabil Saidi. 2020. "Deep Learning for SAR Image Classification Deep Learning for SAR Image Classification." (March).

Jat, Mahesh K., Pradeep Kumar Garg, Susheela Dahiya, and Energy Studies. 2013. "To an Urban Environment International Journal of Image and Data A Comparative Study of Various Pixel- Based Image Fusion Techniques as Applied to an Urban Environment." (February 2016).

Jia, Zhai, Dong Guangchang, Chen Feng, Xie Xiaodan, Qi Chengming, and Li Lin. 2019. "A Deep Learning Fusion Recognition Method Based on SAR Image Data." Procedia Computer Science 147:533-41.

Medasani, Sivasubramanyam, and G. Umamaheswara Reddy. 2017. "Analysis and Evaluation of Speckle Filters for Polarimetric Synthetic Aperture Radar (PolSAR) Data." International Journal of Applied Engineering Research 12(15):4916-27.

Mou, Lichao, Michael Schmitt, Yuanyuan Wang, and Xiao Xiang Zhu. 2017. "A CNN for the Identification of Corresponding Patches in SAR and Optical Imagery of Urban Scenes." (October).

Pottier, Eric. 2011. "Polarimetric SAR Data Analysis." (May).

SAC. 2018. "Overview of NISAR Mission and Airborne L \& S SAR NISAR Mission Concept Overview."

Sun, Zhongchang, $\mathrm{Ru} \mathrm{Xu}$, Wenjie $\mathrm{Du}$, Lei Wang, and Dengsheng Lu. 2019. "High-Resolution Urban Land Mapping in China from Sentinel 1A/2 Imagery Based on Google Earth Engine.” Remote Sensing 11(7):1-22.

Techniques, Hyperspectral. 2018. "Microwave Data Analysis Software Documentation." 0:1-39.

Wang, Zhenyu, Jie Lian, Chunfeng Song, Zhaoxiang Zhang, Wei Zheng, Shaolong Yue, and Senrong Ji. 2019. "SAS: Painting Detection and Recognition via Smart Art System with Mobile Devices.” IEEE Access 7:13556372.

Web, Matec. 2018. “Optical SAR Images Fusion : Comparative Analysis of Resulting Images Data." 01002. 